\title{
APC/C-Cdh1-dependent anaphase and telophase progression during mitotic slippage
}

\author{
Kazuhiro Toda', Kayoko Naito ${ }^{1}$, Satoru Mase ${ }^{1}$, Masaru Ueno ${ }^{1,2}$, Masahiro Uritani ${ }^{1}$, Ayumu Yamamoto ${ }^{1}$ and \\ Takashi Ushimaru ${ }^{1 *}$
}

\begin{abstract}
Background: The spindle assembly checkpoint (SAC) inhibits anaphase progression in the presence of insufficient kinetochore-microtubule attachments, but cells can eventually override mitotic arrest by a process known as mitotic slippage or adaptation. This is a problem for cancer chemotherapy using microtubule poisons.

Results: Here we describe mitotic slippage in yeast bub2 $\Delta$ mutant cells that are defective in the repression of precocious telophase onset (mitotic exit). Precocious activation of anaphase promoting complex/cyclosome (APC/ C)-Cdh1 caused mitotic slippage in the presence of nocodazole, while the SAC was still active. APC/C-Cdh1, but not APC/C-Cdc20, triggered anaphase progression (securin degradation, separase-mediated cohesin cleavage, sisterchromatid separation and chromosome missegregation), in addition to telophase onset (mitotic exit), during mitotic slippage. This demonstrates that an inhibitory system not only of APC/C-Cdc20 but also of APC/C-Cdh1 is critical for accurate chromosome segregation in the presence of insufficient kinetochore-microtubule attachments.

Conclusions: The sequential activation of APC/C-Cdc20 to APC/C-Cdh1 during mitosis is central to accurate mitosis. Precocious activation of APC/C-Cdh1 in metaphase (pre-anaphase) causes mitotic slippage in SAC-activated cells. For the prevention of mitotic slippage, concomitant inhibition of APC/C-Cdh1 may be effective for tumor therapy with mitotic spindle poisons in humans.
\end{abstract}

Keywords: Anaphase promoting complex/cyclosome (APC/C), Bub2, Cdh1, mitotic exit network (MEN), mitotic slippage, Saccharomyces cerevisiae, securin

\section{Background}

The anaphase-promoting complex/cyclosome (APC/C) is an E3 ubiquitin ligase that plays a major role in cell cycle control by targeting substrates for proteasomal degradation. The complex is activated by two WD40 activator proteins, Cdc20/Fizzy/Fzy or Cdh1/Fizzyrelated/Fzr. This destruction is strictly ordered to ensure that cell cycle events are executed in a timely fashion [1-5]. Whereas APC/C-Cdc20 is activated at metaphaseanaphase transition, APC/C-Cdh1 is activated after APC/C-Cdc20 activation. In the budding yeast Saccharomyces cerevisiae, APC/C-Cdh1 is activated from telophase to late $\mathrm{G} 1$ phase [6,7]. The switch from APC/C$\mathrm{Cdc} 20$ to $\mathrm{APC} / \mathrm{C}-\mathrm{Cdh} 1$ is regulated by multiple

\footnotetext{
* Correspondence: sbtushi@ipc.shizuoka.ac.jp

${ }^{1}$ Faculty of Science, Shizuoka University, Shizuoka University, Shizuoka 4228529, Japan

Full list of author information is available at the end of the article
}

mechanisms [5,8-10]: Cyclin B-Cdk1 (cyclin-dependent kinase) inhibits Cdh1 activation in metaphase, but cyclin $\mathrm{B}$ degradation mediated by $\mathrm{APC} / \mathrm{C}$ in late $\mathrm{M}$ phase reduces cyclin $\mathrm{B}-\mathrm{Cdk} 1$ activity, leading to $\mathrm{Cdh} 1$ activation. In addition, APC/C-Cdh1 mediates $\mathrm{Cdc} 20$ degradation, thereby promoting switching from $\mathrm{APC} / \mathrm{C}-\mathrm{Cdc} 20$ to APC/C-Cdh1.

The spindle assembly checkpoint (SAC) ensures faithful chromosome segregation during cell division [11,12]. In the presence of insufficient kinetochore-microtubule attachments, the SAC inhibits anaphase onset by the inhibition of APC/C-Cdc20. The SAC recruits checkpoint proteins, including Mad1, Mad2, Bub1, BubR1 (Mad3 in yeast), Bub3 and Mps1, to unattached kinetochores. As a result, Mad2, BubR1 and Bub3 bind to and suppress APC/C-Cdc20 and form the mitotic checkpoint complex (MCC) [13]. Once all chromosomes have achieved proper kinetochore-microtubule attachment,
Ciomed Central

() 2012 Toda et al; licensee BioMed Central Ltd. This is an Open Access article distributed under the terms of the Creative Commons Attribution License (http://creativecommons.org/licenses/by/2.0), which permits unrestricted use, distribution, and reproduction in any medium, provided the original work is properly cited. 
checkpoint signaling ceases, which is called SAC deactivation or inactivation, and $\mathrm{Mad} 2 / \mathrm{BubR} 1 / \mathrm{Bub} 3$ are released from APC/C-Cdc20. It allows active APC/C$\mathrm{Cdc} 20$ to drive cells into anaphase by inducing the degradation of securin and cyclin B. The degradation of securin permits sister-chromatid separation, and the destruction of cyclin B reduces Cdk1 activity. In contrast to the molecular mechanisms of the SAC activation, those of SAC deactivation are poorly understood $[14,15]$.

Microtubule targeted drugs are of clinical importance in the successful treatment of a variety of human cancers because they activate the SAC and induce mitotic arrest that leads to apoptotic cell death [16]. However, in the continued presence of conditions that normally keep the SAC active, some cells escape from mitosis, resulting in tetraploid cells $[16,17]$. This phenomenon is termed mitotic slippage or adaptation. This process is largely responsible for the failure to efficiently block tumor progression. Mitotic slippage depends on progressive degradation of cyclin $\mathrm{B}$, while the SAC is active, indicating that mitotic slippage occurs through the overriding of activated SAC signaling [18,19]. Mitotic exit occurs once cyclin B-Cdk1 activity has decreased below a critical threshold required to maintain a mitotic state [18]. In addition to cyclin B, other mitotic APC/C substrates, including securin, are also degraded during mitotic slippage, and a double knockdown of Cdc20 and Cdh1 prevents the degradation of $\mathrm{APC} / \mathrm{C}$ substrates during mitotic slippage [20]. These findings indicate that $\mathrm{APC} / \mathrm{C}$ is critical for mitotic slippage. However, which protein does mitotic slippage require, $\mathrm{Cdc} 20$ or Cdh1? Furthermore, how can APC/C be activated, although the SAC is active? The degradation of Cyclin $\mathrm{A}$ and NIMA-related kinase 2A (Nek2A) in early mitosis is dependent on $\mathrm{APC} / \mathrm{C}-\mathrm{Cdc} 20$, and this process is not inhibited by the SAC [21]. While the SAC-dependent substrate cyclin $\mathrm{B}$ requires $\mathrm{Cdc} 20$ for recruitment to $\mathrm{APC} / \mathrm{C}, \mathrm{Nek} 2 \mathrm{~A}$ can bind the $\mathrm{APC} / \mathrm{C}$ in the absence of Cdc20 [22]. Thus, the SAC suppresses the degradation of most, but not all, substrates of APC/C-Cdc20. However, degradation of cyclin A and Nek2A does not trigger metaphase-anaphase transition and mitotic slippage. It is unclear how mitotic exit (telophase onset) can be initiated in metaphase-arrested cells during mitotic slippage; less attention has been paid to how anaphase is executed during mitotic slippage.

In budding yeast, mitotic slippage-like phenomena have been reported, but they are relatively ill-defined, as compared with mammalian cells, because the SAC status is obscure. It is important to determine the SAC status during mitotic slippage (and slippage-like phenomena), in order to distinguish mitotic slippage that overrides the activated SAC from events caused by SAC deactivation. Mitotic exit accompanied by securin degradation, sister-chromatid separation and nuclear division was found after treatment of the wild-type yeast cells with the microtubule depolymerizer benomyl but not with nocodazole [23]. It is unknown whether these phenomena found in the presence of benomyl are indeed mitotic slippage, because the SAC status has not been characterized. Interestingly, mitotic slippage (or slippage-like phenomena) is prominently observed in mutant cells deficient in the budding uninhibited by benzimidazole (BUB) 2 gene in the presence of nocodazole. Among BUB proteins, whereas Bub1 and Bub3 are components of the SAC, Bub2 is an inhibitor of the mitotic exit network (MEN) that promotes anaphase-telophase transition [8-10,24,25]. Although bub2 $\Delta$ cells exhibit an intact SAC, they fail to arrest in metaphase and exit from mitosis in the presence of nocodazole, leading to cell death [24,26-28]. In addition, bub2 $\Delta$ cells cannot effectively arrest at metaphase when the SAC is activated by $M A D 2$ overexpression [23].

Nocodazole-treated bub2 $\Delta$ cells exhibit securin degradation, sister-chromatid separation (indexes of anaphase progression) and rebudding (an index of telophase progression and mitotic exit) [24,26,27]. Thus, anaphase and telophase progression occurs in nocodazole-treated bub $2 \Delta$ cells. A mutation in the MEN factor Tem1 suppresses bub2 $\Delta$-induced sister-chromatid segregation [28]. It suggests that precocious activation of the MEN causes mitotic slippage, but the molecular mechanism responsible is largely unknown. Although securin degradation and sister-chromatid separation are normally mediated by APC/C-Cdc20 at anaphase onset, these events found in nocodazole-treated $b u b 2 \Delta$ cells are not repressed by a $c d c 20-3$ mutation at a restrictive temperature [29]. These findings indicate that anaphase progression in nocodazole-treated $b u b 2 \Delta$ cells occurs independently of APC/C-Cdc20. On the other hand, sister-chromatid separation in nocodazole-bub2 $\Delta$ cells is suppressed by a lack of the APC/C core subunit Cdc26 [27], suggesting that $\mathrm{APC} / \mathrm{C}$ itself is required for $b u b 2 \Delta$ mediated anaphase progression. Thus, the bub2 $\Delta$ stain might be a useful model for mitotic slippage. We show herein that nocodazole-treated bub2 $\Delta$ cells override the active SAC-mediated metaphase arrest and cause securin degradation, sister-chromatid separation and mitotic exit and that $\mathrm{APC} / \mathrm{C}-\mathrm{Cdh} 1$ is critical for mitotic slippage.

\section{Results}

Precocious activation of the MEN induces mitotic slippage We hypothesized that mitotic slippage in bub2 $\Delta$ cells is caused by activation of the mitotic exit system in metaphase-arrested cells. To describe anaphase and telophase progression during mitotic slippage in bub2 $\Delta$ cells, we followed sister-chromatid separation (an index of 


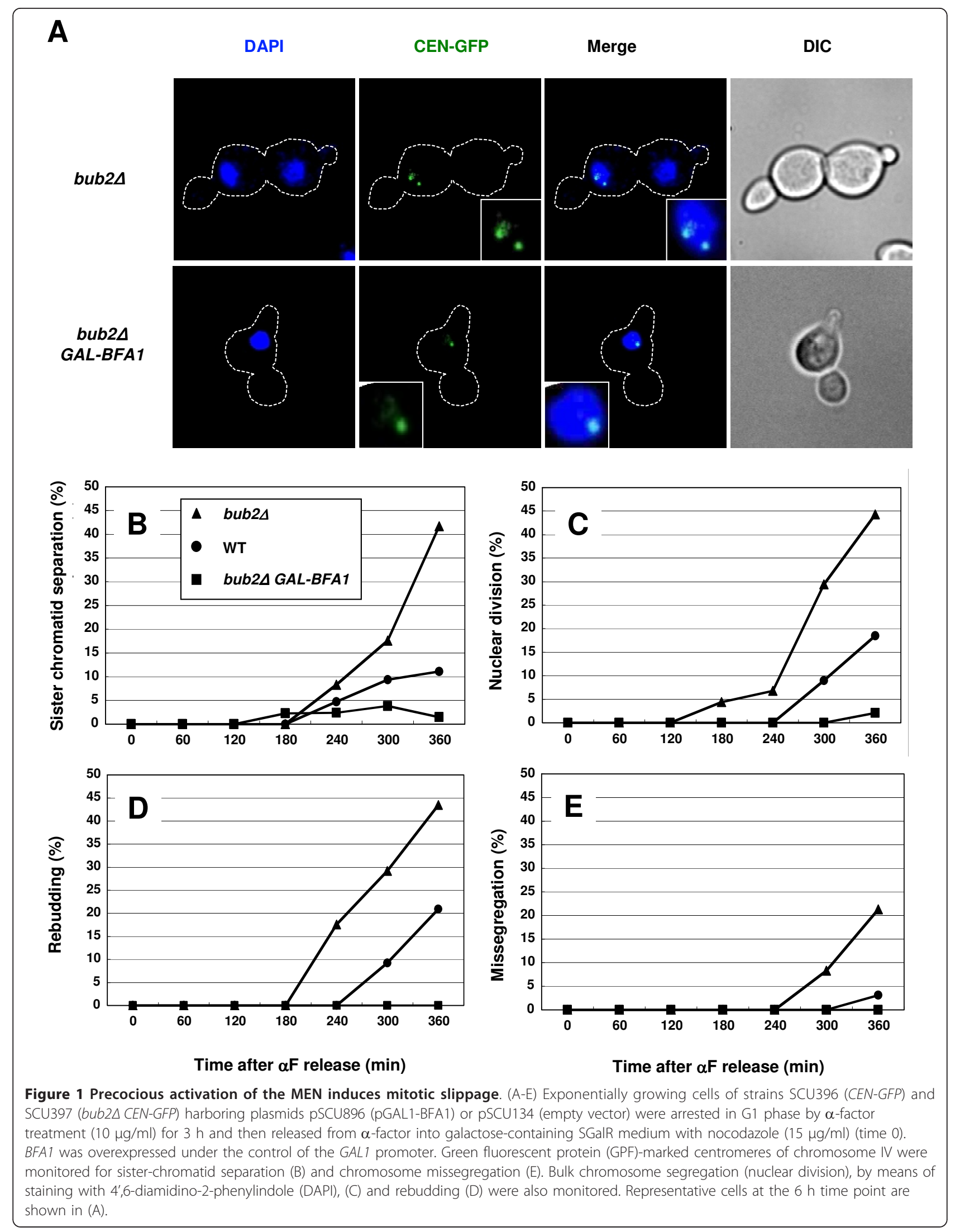


anaphase onset) using the lacO/lacI system [26] and new bud formation (rebudding; an index of telophase progression and mitotic exit). Cells were released from $\alpha$-factor (G1 arrest) into a nocodazole-containing medium. The bub2 $\Delta$ cells gradually overrode metaphase arrest and showed sister-chromatid separation and rebudding (Figures 1A, B and 1D), as described previously $[24,26]$. We observed frequent chromosome missegregation in the bub2 $\Delta$ cells (Figures $1 \mathrm{~A}, \mathrm{E}$ ). When the SAC is deactivated after establishment of proper kinetochore-microtubule attachment, chromosomes could be accurately segregated. In contrast, chromosome missegregation might frequently occur in cells during mitotic slippage, because under these conditions anaphase progression occurs even when there are improper/insufficient kinetochore-microtubule attachments. Chromosome missegregation observed here supports the occurrence of mitotic slippage in nocodazole-treated bub2 $2 \Delta$ cells. In contrast, a smaller portion of wild-type cells showed sister-chromatid separation and rebudding under the same conditions. Thus, Bub2 is critical for the prevention of mitotic slippage.

Given the role of Bub2, it is most likely that precocious MEN activation occurs in bub2 $2 \Delta$ cells, leading to mitotic slippage. To assess this idea, we examined whether overexpression of the MEN inhibitor Bfal cancels mitotic slippage in bub2 $\Delta$ cells. This was indeed the case: sister-chromatid separation and rebudding was completely repressed by BFA1 overexpression (Figures 1A-C, bub2 $\triangle$ GAL-BFA1). This demonstrated that MEN activation causes anaphase and telophase onset during mitotic slippage. It was also reported that a mutation in the MEN factor Tem1 suppresses bub2 $\Delta$-induced sisterchromatid segregation [28].

Because nuclear division (an index of anaphase progression) is dependent on spindle microtubules, it is inhibited when microtubules are completely abrogated. However, nuclear division in the bub2 $\Delta$ cells treated with nocodazole (LKT Laboratories, Lot No. QJ1275) was identified, although no detectable microtubules were found in the indirect immunoflorescence assay (data not shown). This finding suggested that there were imperceptible microtubules causing nuclear division. However, it was noteworthy that the SAC was still active under these conditions (see below). These findings indicated that nocodazole continues to activate the SAC sufficiently and that the phenomena found in bub2 $2 \Delta$ cells here are caused by mitotic slippage but not SAC deactivation/inactivation.

We also examined mitotic slippage of bub2 $\Delta$ cells when the SAC gene MAD2 was overexpressed. MAD2 overexpression causes SAC activation-mediated metaphase arrest, but during a long-term treatment cells override metaphase arrest and cause cell proliferation, although profiles of sister-chromatid separation and nuclear division, chromosome missegregation during mitotic slippage were not described [23]. When MAD2 was overexpressed for $6 \mathrm{~h}$, rebudding (mitotic exit) was frequently found in bub2 $\Delta$ cells, as compared with wildtype cells (Additional file 1), which was consistent with the finding that cell proliferation was promoted by the bub2d mutation [23]. Furthermore, it was found that sister-chromosome segregation and nuclear division were also prominent in bub2 $\Delta$ cells (Additional file 1). Thus, both nocodazole treatment and MAD2 overexpression similarly caused mitotic slippage in bub2 $\Delta$ cells. In contrast, chromosome missegregation in $M A D 2$-overexpressing bub2 $\triangle$ cells was not detectable (data not shown), which was probably because in this case microtubules were intact and a proper kinetochoremicrotubule attachment was established.

\section{APC/C-Cdh1 is critical for chromosome separation during mitotic slippage}

Sister-chromatid separation is triggered by APC/C$\mathrm{Cdc} 20$ at normal anaphase onset, but $\mathrm{APC} / \mathrm{C}-\mathrm{Cdc} 20$ is suppressed by the SAC in nocodazole-treated cells. This suggests that APC/C-Cdc20 is not involved in mitotic slippage. In fact, it was reported that sister-chromatid separation in nocodazole-treated bub2 $2 \Delta$ cells is not suppressed by a temperature sensitive $c d c 20-3$ mutation [29]. We again found that Cdc20 depletion did not suppress sister-chromatid separation in the nocodazoletreated bub2 $\Delta$ cells (Figure 2A, B). Rebudding was also not suppressed by $\mathrm{Cdc} 20$ depletion. This finding demonstrated that $\mathrm{Cdc} 20$ is not required for mitotic slippage. Consistently, it was reported that rebudding of nocodazole-treated $b u b 2 \Delta$ cells was not suppressed by a temperature sensitive $c d c 20-1$ mutation [30].

We suspected that precocious MEN activation in nocodazole-treated bub $2 \Delta$ cells causes activation of APC/C-Cdh1, leading to mitotic slippage. Indeed, a lack of Cdh1 markedly repressed sister-chromatid separation and nuclear division in nocodazole-treated bub2 $2 \Delta$ cells (Figure 2A, B). These observations clearly indicated that $\mathrm{APC} / \mathrm{C}-\mathrm{Cdh} 1$, but not $\mathrm{APC} / \mathrm{C}-\mathrm{Cdc} 20$, is responsible for MEN-mediated anaphase progression. In contrast, rebudding (mitotic exit) was not suppressed by $\mathrm{CDH} 1$ deletion, probably because the CDK inhibitor Sic1 induced by the MEN also contributes to the repression of CDK activity and is sufficient for mitotic exit in $c d h 1 \Delta$ cells, as in normal mitosis.

APC/C-Cdh1-mediated securin degradation is required for sister-chromatid separation during mitotic slippage

Sister-chromatid separation requires securin degradation. We examined securin degradation in nocodazoletreated bub2A cells. The yeast securin Pds1 is 


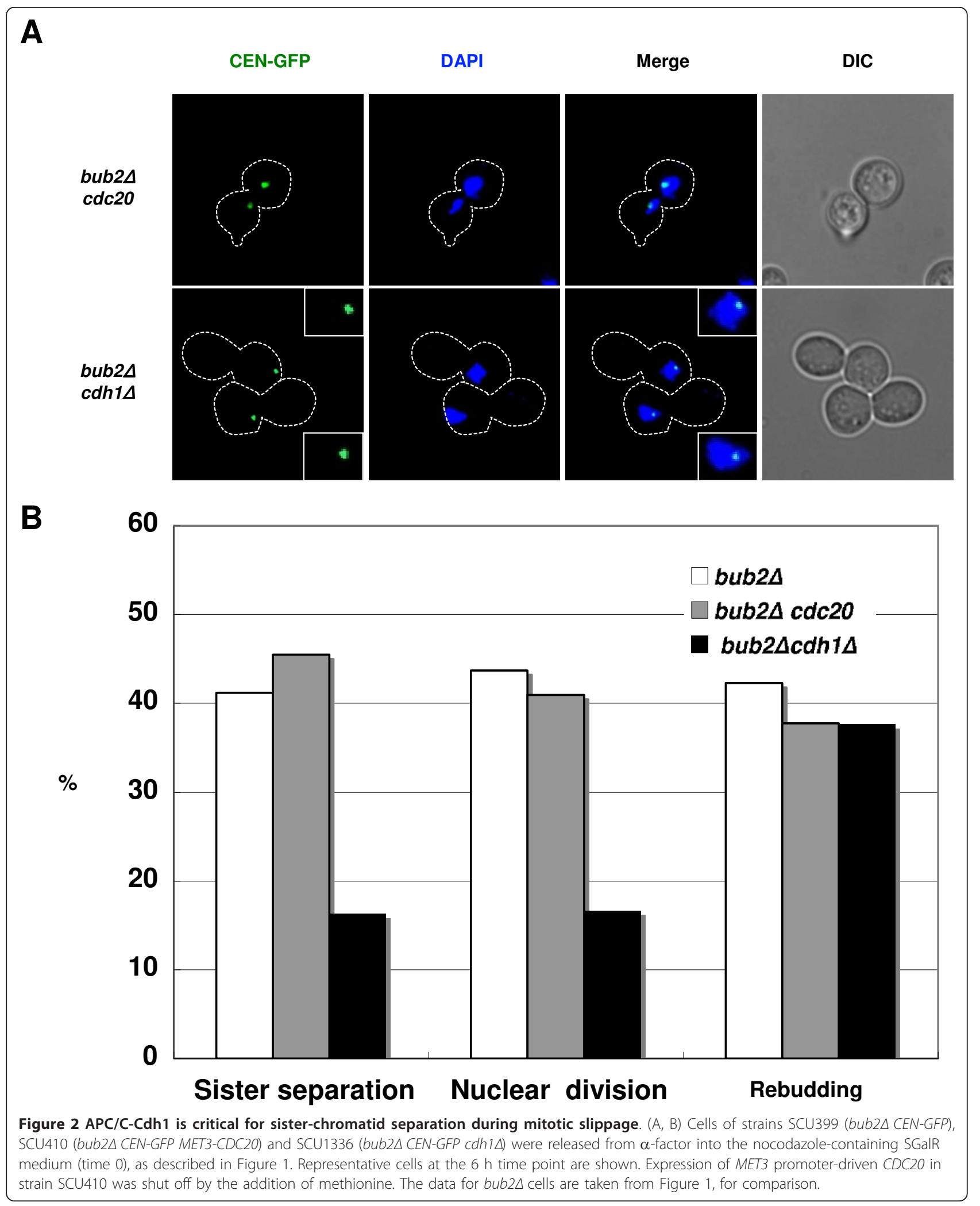


phosphorylated by CDK, and it was often detected as two bands in western blotting analysis $[31,32]$. Securin degradation was repressed in the presence of nocodazole by the SAC in wild-type cells, but it occurred in bub2 $\Delta$ cells [27] (Figure 3A, WT and bub2 4 ). Because sisterchromatid separation in nocodazole-treated bub2 $\Delta$ cells was APC/C-Cdh1 dependent (Figure 2), it would be expected that $\mathrm{APC} / \mathrm{C}-\mathrm{Cdh} 1$ is required for securin degradation during mitotic slippage in nocodazole-treated bub2 $\Delta$ cells. In the normal cell cycle, it was suggested that APC/C-Cdh1 mediates securin degradation after telophase onset (see "Discussion"). Indeed, securin degradation was largely suppressed by the loss of Cdh1 in bub2 $\Delta$ cells (Figure $3 \mathrm{~A}$ ): bub2 $\Delta$ cdh1 $\Delta$ cells reproducibly had more securin at G1 phase (0 time point). This may result from the repression of securin degradation in the previous mitosis. Thereafter, securin levels were approximately equal between $b u b 2 \Delta$ and $b u b 2 c d h 1 \Delta$ cells 1 hour after G1 release, and then securin degradation was obvious only in bub2 2 cells. Thus, APC/CCdh1 triggered securin degradation during mitotic slippage.

Next, we examined whether this securin degradation during mitotic slippage is required for sister-chromatid separation. APC/C-Cdh1 targets securin through D- and KEN boxes [33,34]. To test this, we ectopically expressed a non-degradable securin mutant devoid of both D- and KEN boxes (securin-dkb) [34]. Securin-dkb strongly repressed sister-chromatid separation (Figures $3 \mathrm{~B}, \mathrm{C})$. In contrast, as expected, expression of a securin mutant lacking only the D-box (securin- $d b$ ) repressed sister-chromatid separation less effectively. These findings demonstrated that APC/C-Cdh1-mediated securin degradation is a prerequisite for sister-chromatid separation during mitotic slippage in nocodazole-treated bub2 2 cells.

\section{Separase executes sister-chromatid separation and nucleolar segregation during mitotic slippage}

After APC/C-Cdc20-dependent securin degradation at normal anaphase onset, the liberated separase cleaves the subunit of cohesin Scc1, allowing sister-chromatid separation [35]. On the other hand, cohesin removal from chromosomes is also performed via another route; in higher eukaryotes, cohesins are largely dissociated from the chromosome arms in prophase in a separaseindependent manner (called the prophase pathway) $[36,37]$. The prophase pathway has not yet been described in yeast, but recent reports suggested the occurrence of separase-independent cohesin removal and chromosome separation [38,39]. We examined whether separase-mediated Scc1 cleavage is required for sister-chromatid separation during mitotic slippage with the use of Scc1-RRDD, a non-cleavable mutant version of Scc1 [40]. Ectopic expression of Scc1-RRDD drastically suppressed sister-chromatid separation (Figures 4A, B). This clearly demonstrated that sister-chromatid separation requires separase-mediated Scc1 cleavage during mitotic slippage. Overall, APC/C-Cdh1 triggers securin degradation, separase liberation and then cohesin cleavage, causing sister-chromatid separation during mitotic slippage in nocodazole-treated bub2 $\Delta$ cells.

In normal early anaphase, the liberated separase also causes nucleolar segregation in a manner independent of its protease activity [41-43]. The nucleolar segregation into mother and daughter cells was observed, together with nuclear division during mitotic slippage in the bub2 2 cells (Figure $4 C$ ). This indicates that proteaseindependent action of separase is also promoted during mitotic slippage.

\section{The SAC is active during mitotic slippage}

In mammalian cells, mitotic slippage occurs with SAC being still active and cells override the SAC-mediated metaphase arrest (see "Introduction"). To test whether the SAC is active in the present case, we examined kinetochore localization of Mad2, an index of SAC activation (Figure 5A). We observed GFP-tagged Mad2 signals on the kinetochores, which were marked by RFP (red fluorescent protein)-tagged Mtw1 (a kinetochore protein). We observed colocalization of Mad2 and Mtw1 signals in nocodazole-treated bub2 $\Delta$ cells, as also observed in nocodazole-treated wild-type cells (Figures $5 \mathrm{~A}, \mathrm{~B})$, indicating that the SAC is still active in the bub2 $\Delta$ cells, like in the wild-type cells. This demonstrates that SAC deactivation does not cause sister-chromatid separation, securin degradation and rebudding in the nocodazole-treated $b u b 2 \Delta$ cells. Cell images were captured with single $\mathrm{Z}$-axis sections using a microscope. Because the size of the kinetochore is considerably smaller than the cell, $100 \%$ of the signals of Mad2-GFP on the kinetochores were not detectable even if all Mad2 signals were localized on the kinetochores in all cells. It was noteworthy that both mother and daughter cells had one Mad2 dot signal on the kinetochore each in some bub2 $\Delta$ cells (Figures 5A, B). Because kinetochore segregation to both mother and daughter cells indicates anaphase progression in these cells, these findings demonstrated that anaphase progression occurred with an active SAC in nocodazole-treated bub2 $\Delta$ cells and that the cells overrides metaphase arrest by the activated SAC. Thus, precocious APC/C-Cdh1 activation overrides $\mathrm{SAC}$-mediated metaphase arrest and causes mitotic slippage.

\section{Ectopic activation of Cdh1 causes mitotic slippage}

To test the idea that precocious activation of APC/CCdh1 causes mitotic slippage, we examined whether 
A
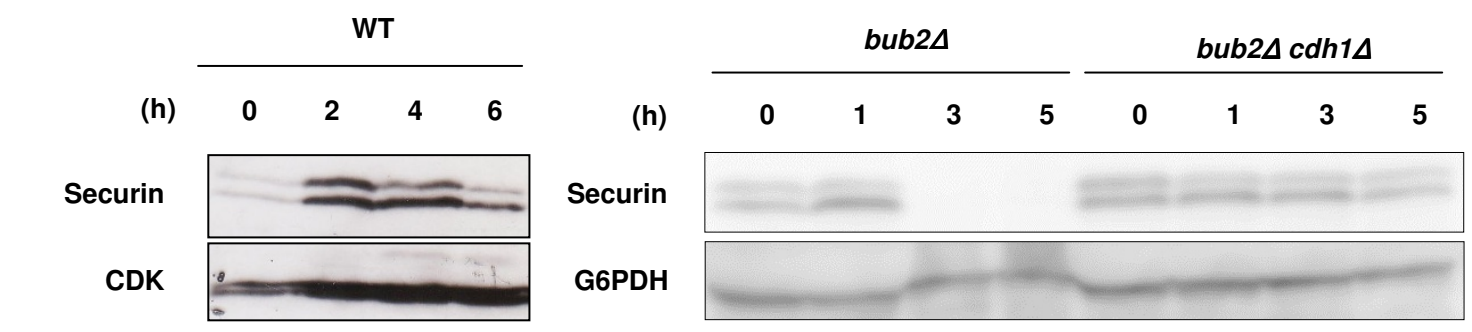

B

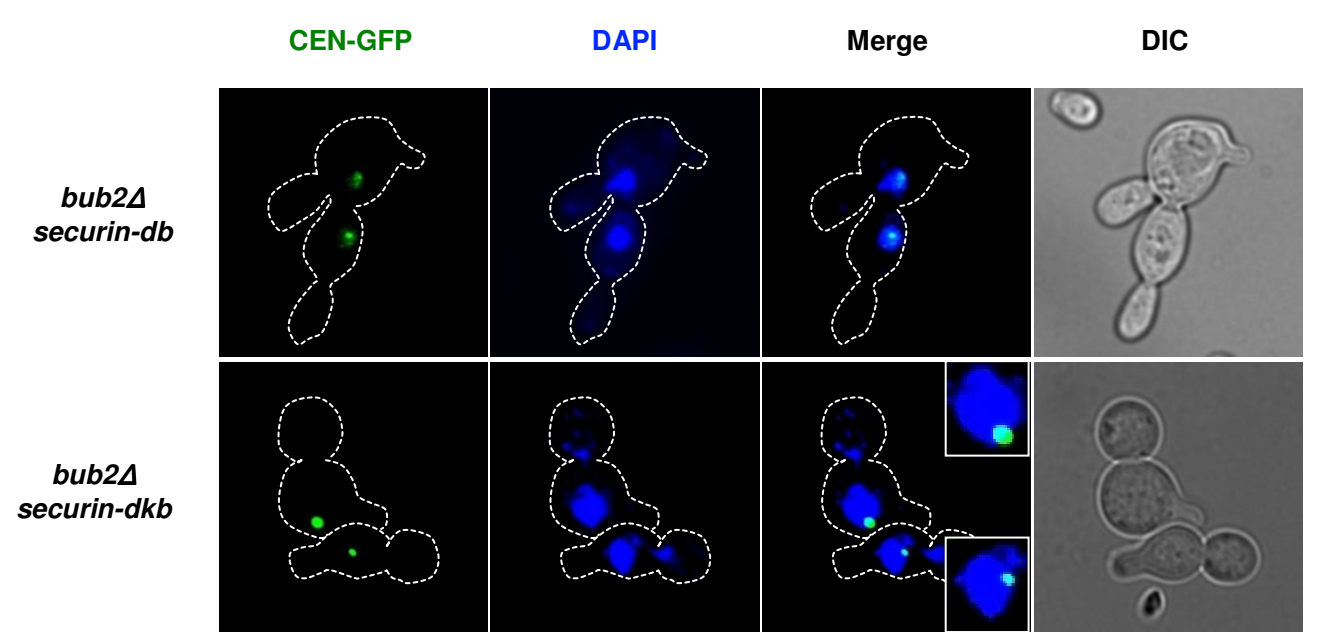

C

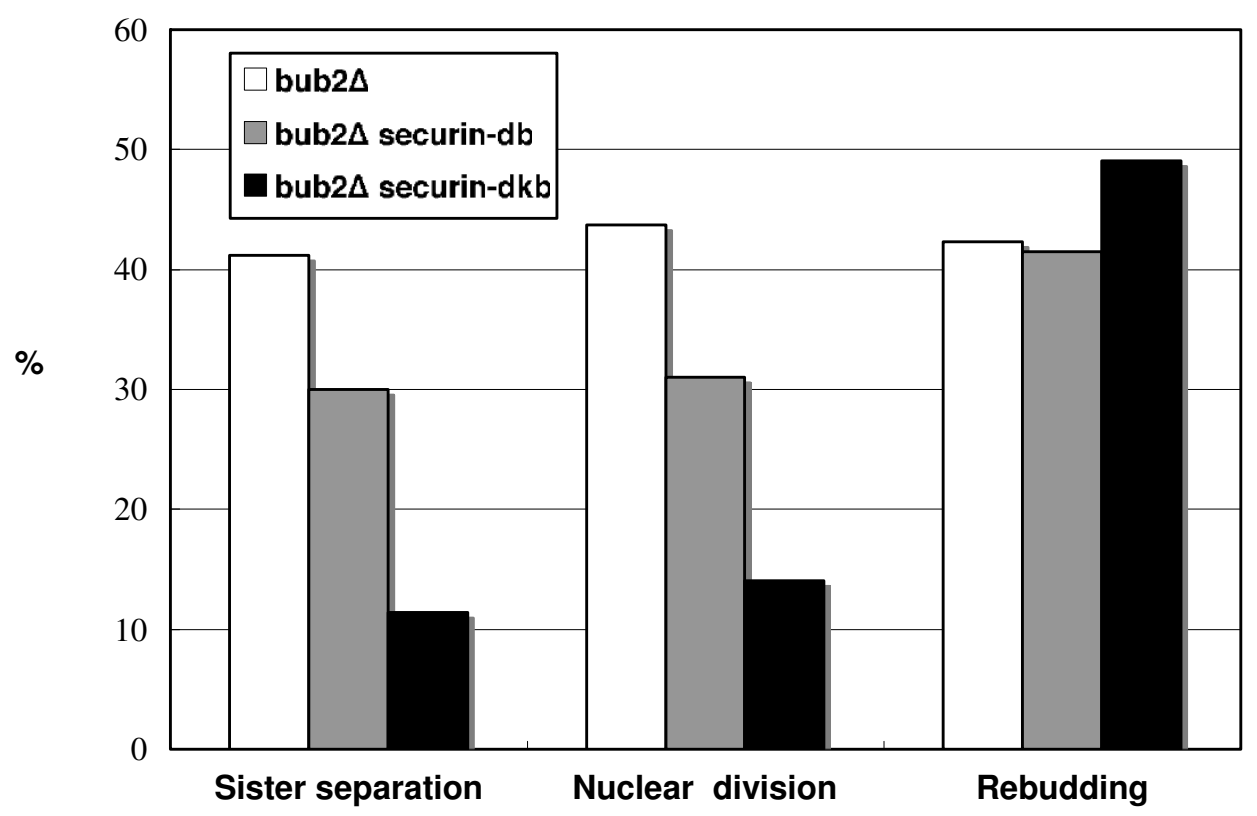

Figure $3 \mathrm{APC} / \mathrm{C}-\mathrm{Cdh} 1-$ mediated securin degradation is required for sister-chromatid separation during mitotic slippage. (A) Cells of

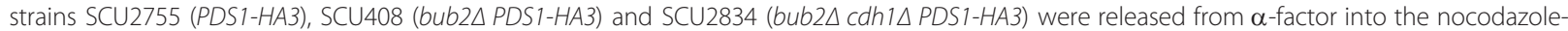
containing medium (time 0), as described in Figure 1. HA-tagged securin (Pds1-HA3) was detected by western blotting analysis using an anti-HA antibody. CDK and glucose-6-phosphate dehydrogenase (G6PDH) were used as the loading controls. (B, C) Cells of strain SCU396 (bub2 $\triangle$ CEN-GFP) with plasmids pSCU1212 (pGAL1-pds1-db) or pSCU1214 (pGAL1-pds1-dkb) were released from $\alpha$-factor into nocodazole-containing SGalR medium (time 0), as described in Figure 1. Representative cells at the $6 \mathrm{~h}$ time point are shown. The data for bub2 $\Delta$ cells are taken from Figure 1. 


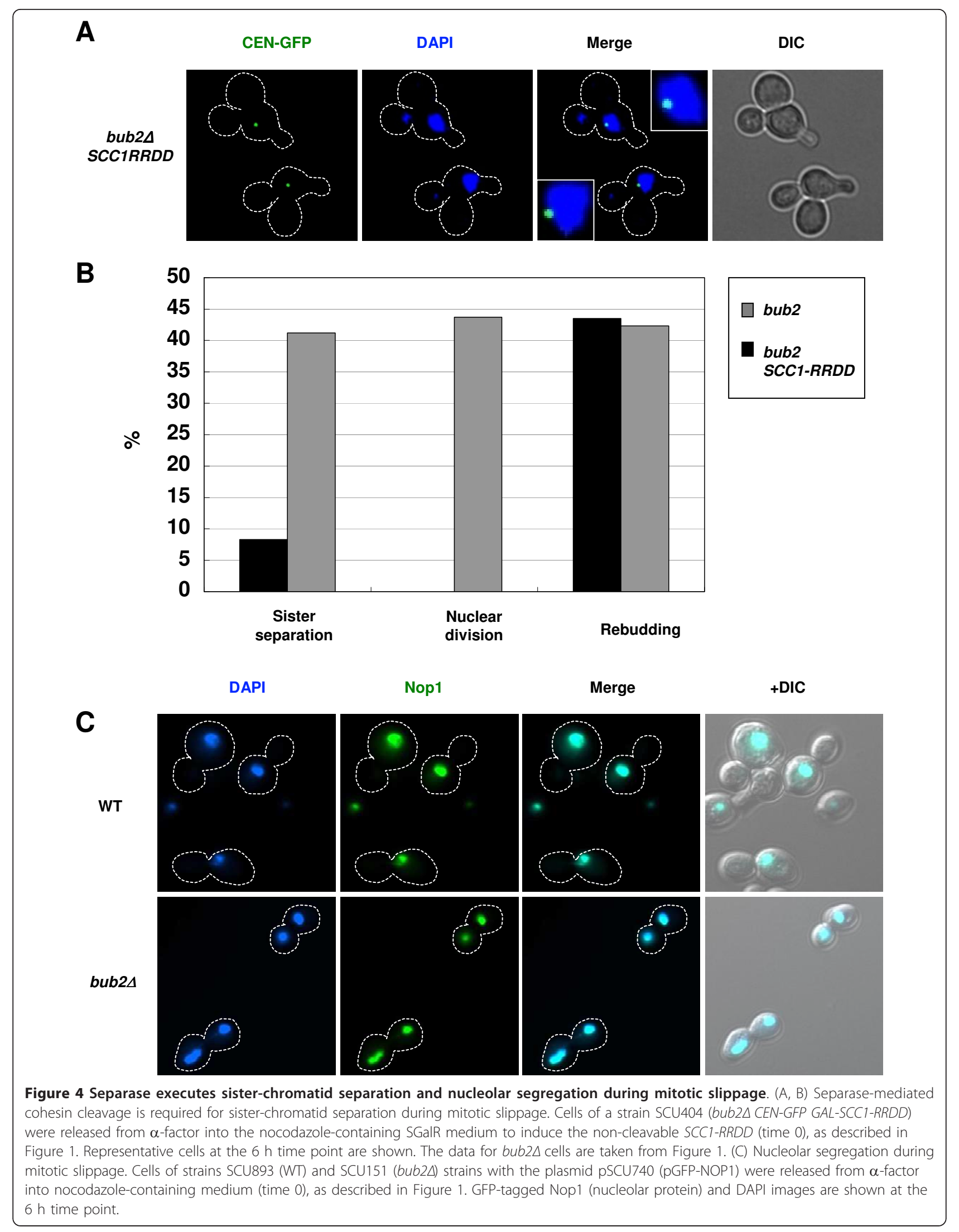




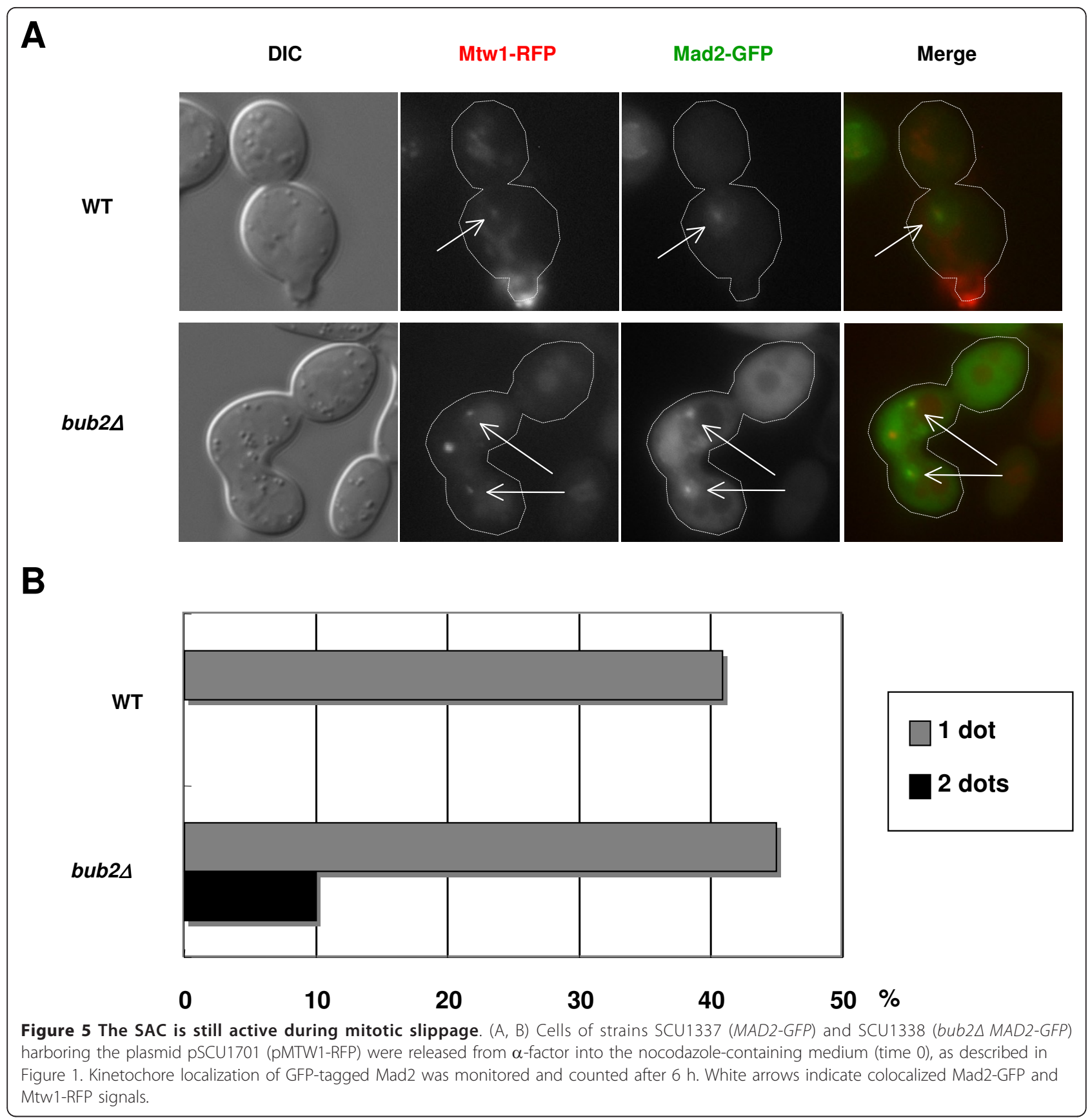

ectopic activation of Cdh1 similarly causes mitotic slippage. Cells were released from $\alpha$-factor (G1 phase) to a medium containing nocodazole and galactose to overexpress $C D H 1$. $C D H 1$ overexpression brought about no securin accumulation, sister-chromatid separation or nuclear division with chromosome missegregation (Figures $6 \mathrm{~A}-\mathrm{C}$ ). Rebudding was lowered by $\mathrm{CDH1}$ overexpression under these conditions for an unknown reason (Figure 6B), whereas $\mathrm{CDH1}$ overexpression in metaphase-arrested cells treated with nocodazole caused securin degradation and rebudding (Figure 6D and data not shown). Thus, ectopic activation of APC/C-Cdh1 caused mitotic slippage.

Cdc14 phosphatase, which antagonizes CDK, promotes APC/C-Cdh1 activation and mitotic exit in telophase $[5,9,41]$. CDC14 overexpression from $\mathrm{G} 1$ phase induced securin degradation (Figure $6 \mathrm{C}$ ) but inhibited G1/S progression [44] (data not shown). Hence, we overexpressed CDC14 in metaphase-arrested cells treated with nocodazole. CDC14 overexpression promoted securin degradation and sister-chromatid separation (Figures 6D-F). However, both of these events were repressed in $c d h 1 \Delta$ 


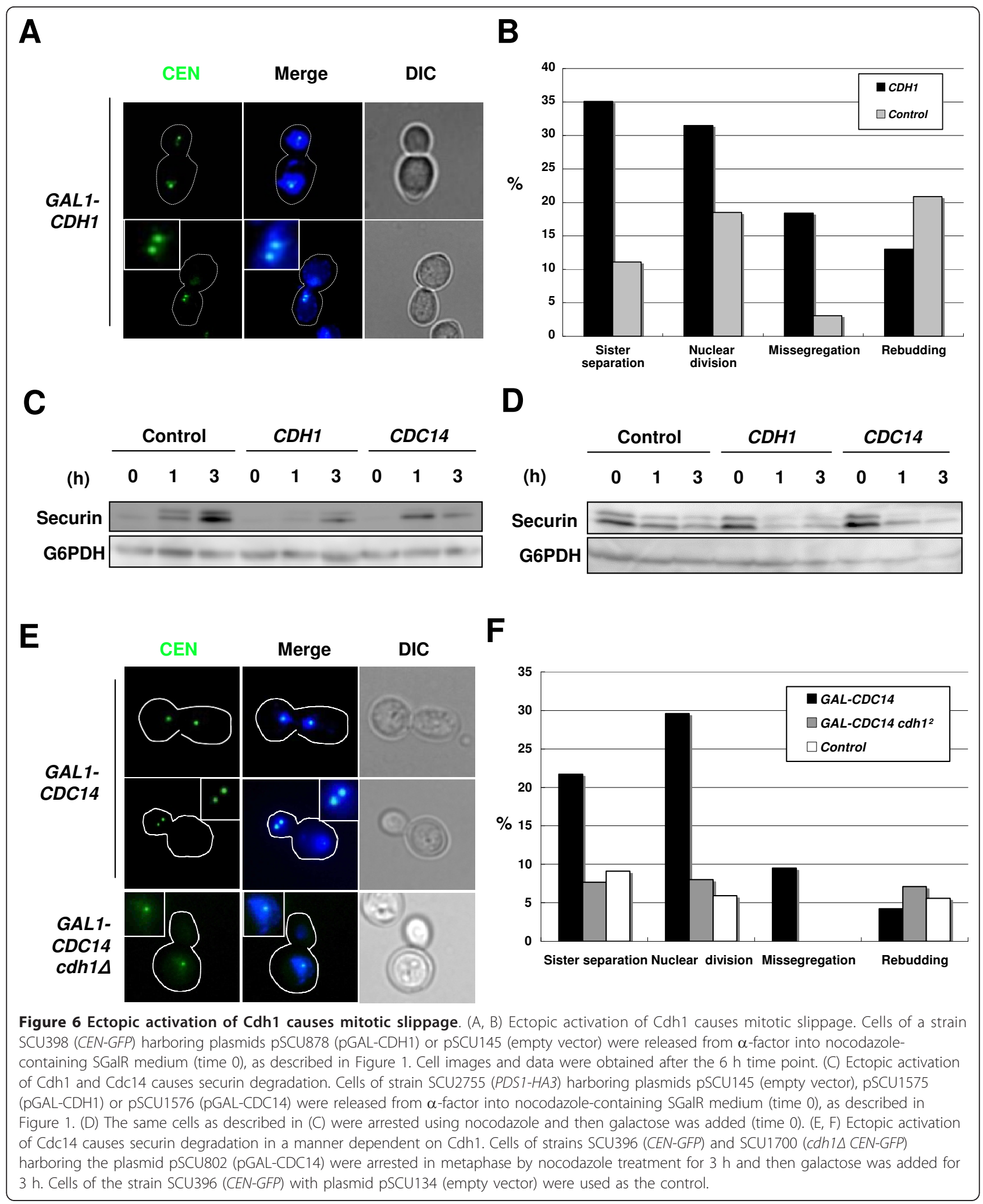


cells (Figure 6E, F). These findings indicated that ectopic activation of Cdc14 causes mitotic slippage via APC/CCdh1. Consistent with the previous report that $C D C 14$ overexpression promotes mitotic exit but represses budding in the next $S$ phase, because of the counteraction of Cdc14 against CDK-mediated phosphorylation [44], no promotion of rebudding in the next $\mathrm{S}$ phase by $C D C 14$ overexpression was observed (Figure 6F). Overall, these findings in Cdh1- and Cdc14-overexpressing cells supported the notion that precocious activation of APC/ $\mathrm{C}-\mathrm{Cdh} 1$ in pre-anaphase triggers mitotic slippage.

\section{Discussion}

Precocious activation of APC/C-Cdh1 in metaphase causes mitotic slippage

While APC/C-Cdc20 is activated at metaphase-anaphase transition, APC/C-Cdh1 is activated at anaphase-telophase transition [6,7] (Figure 7A). The switch from

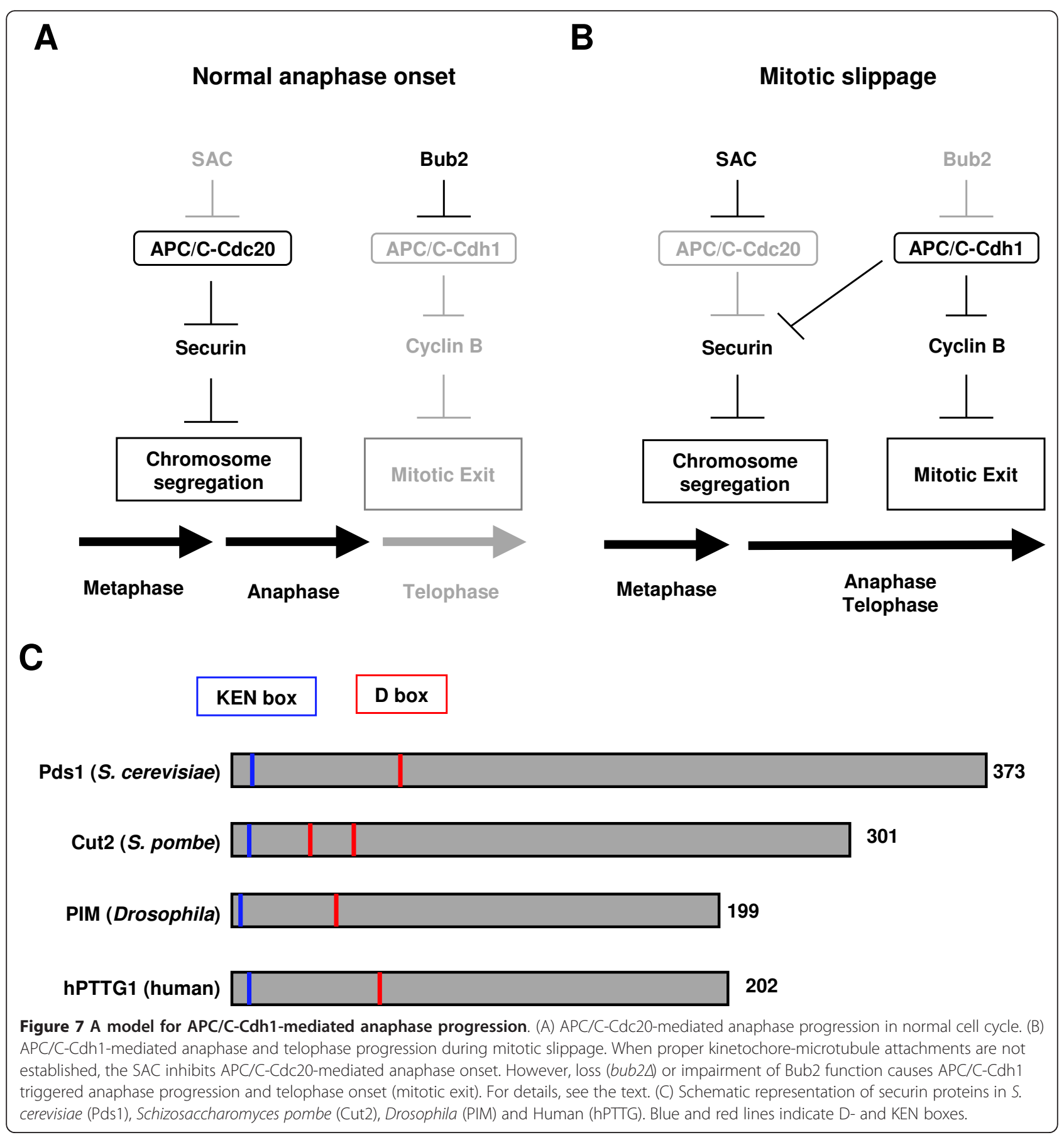


$\mathrm{APC} / \mathrm{C}-\mathrm{Cdc} 20$ to $\mathrm{APC} / \mathrm{C}-\mathrm{Cdh} 1$ is regulated by multiple mechanisms $[5,8-10]$. This sequential activation is thought to be the heart of accurate mitosis, but this notion has not yet been fully tested. This study showed that precocious activation of APC/C-Cdh1 in metaphase (pre-anaphase) caused mitotic slippage in nocodazoletreated cells and that APC/C-Cdh1, instead of APC/CCdc20, could trigger anaphase progression, in addition to telophase progression (Figure 7B).

$\mathrm{APC} / \mathrm{C}-\mathrm{Cdh} 1$-mediated anaphase progression during mitotic slippage had two prominent features. First, APC/C-Cdh1-mediated anaphase progression brought about chromosome missegregation, because APC/CCdh1 is not inhibited by the SAC in the presence of inappropriate kinetochore-microtubule attachments; therefore, APC/C-Cdh1-mediated securin degradation results in chromosome missegregation. This demonstrated that an inhibitory system not only of APC/CCdc20 but also of APC/C-Cdh1 is critical for accurate chromosome segregation in the presence of insufficient kinetochore-microtubule attachments.

Second, APC/C-Cdh1 simultaneously starts anaphase and telophase from metaphase. $\mathrm{APC} / \mathrm{C}-\mathrm{Cdc} 20$ recognizes the D-box of a relatively limited umber of targets (the important targets are only cyclin $\mathrm{Clb} 5$ and securin Pds 1 in budding yeast) [45], whereas APC/C-Cdh1 recognizes various motifs on numerous targets (A-, O-, CRY and GxEN boxes, in addition to D- and KEN boxes) [2,46-48]. Namely, APC/C-Cdh1 could target substrates for APC/CCdc20, which allowed simultaneous onsets of anaphase and telophase. APC/C-Cdh1 targets securin Pds1 in a manner dependent on D- and KEN boxes in vitro [33,34], and ectopically expressed Pds1 in G1 phase was degraded in a manner dependent on APC/C-Cdh1 [49]. These findings suggested that $\mathrm{APC} / \mathrm{C}-\mathrm{Cdh} 1$ mediates securin degradation from telophase to $\mathrm{G} 1$ phase in vivo. If $\mathrm{APC} / \mathrm{C}$ $\mathrm{Cdh} 1$ becomes activated abnormally in metaphase, it can target securin, leading to sister-chromatid separation (Figure 7B). This study clarifies these abnormal aspects of precocious APC/C-Cdh1 activation in metaphase cells and emphasizes that sequential activation of APC/C-Cdc20-to$\mathrm{APC} / \mathrm{C}-\mathrm{Cdh} 1$ is critical for mitosis.

Deregulation of APC/C-Cdh1 in other cell phases brings about different outputs. $\mathrm{CDH} 1$ overexpression in asynchronized cells leads to elongated buds, G2 phase arrest, and 4C DNA content in some cells $[6,7,50]$. Precocious activation of APC/C-Cdh1 in G2 phase targets proteins that are required for separation of the spindle pole body (SPB, yeast centrosome), the BimC family kinesins Cin8/Eg5 and Kip1 and the interpolar microtubule midzone protein Ase1 [51,52]. Thus, deregulation of Cdh1 activity compromises genome transmission in various ways and timely activation and inactivation of APC/C$\mathrm{Cdh} 1$ are pivotal for accurate genome transmission.

\section{APC/C-Cdh1-mediated mitotic slippage in other organisms}

In fission yeast, the septation initiation network (SIN), a signaling pathway homologous to the MEN, coordinates mitosis and cytokinesis [8,25,53,54]. Cdc16 (Bub2 ortho$\log$ ) acts as a negative factor of the SIN, and $c d c 16$ mutant cells undergo cytokinesis in the presence of the microtubule destabilizer thiabendazole [55-57]. This suggests that SIN-mediated APC/C-Cdh1/Ste9 activation causes mitotic slippage. In addition, the fission yeast securin Cut2 also has D- and KEN boxes (Figure 7C). We postulate that APC/C-Cdh1/Ste9-mediated securin degradation and sister-chromatid separation is promoted during mitotic slippage in fission yeast.

In mammalian mitosis, $\mathrm{APC} / \mathrm{C}-\mathrm{Cdc} 20$ and $\mathrm{APC} / \mathrm{C}-$ $\mathrm{Cdh} 1$ are sequentially activated $[3,5,58]$. Mitotic slippage depends on progressive degradation of cyclin $\mathrm{B}$ with the SAC active $[18,19]$. This suggests that APC/C-Cdh1, but not $\mathrm{APC} / \mathrm{C}-\mathrm{Cdc} 20$, is also involved in mitotic slippage in mammalian cells. Cdc20 and Cdh1 target securin in a manner dependent on D/KEN-boxes [59,60] (see Figure $7 \mathrm{C})$. This suggests that precocious activation of APC/CCdh1 similarly causes securin degradation and sisterchromatid separation during mitotic slippage in mammalian cells. In fact, deregulation of Cdh1 in pre-anaphase results in premature securin degradation and sister-chromatid separation $[59,61]$. The present study predicts that for prevention of mitotic slippage, concomitant inhibition of APC/C-Cdh1 may be effective for tumor therapy with mitotic spindle poisons in humans.

\section{Conclusions}

The sequential activation of APC/C-Cdc20-to-APC/C$\mathrm{Cdh} 1$ during mitosis is critical for accurate mitosis. Precocious activation of APC/C-Cdh1 in metaphase (preanaphase) causes mitotic slippage in microtubule poison-treated cells. For prevention of mitotic slippage, concomitant inhibition of APC/C-Cdh1 may be effective for tumor therapy with mitotic spindle poisons in human.

\section{Methods}

\section{Strains, plasmids, media and materials}

S. cerevisiae strains and plasmids used are listed in Tables 1 and 2. Glucose-containing YPAD (YPD containing $0.01 \%$ adenine) and synthetic minimal medium (SD) complemented with the appropriate nutrients for plasmid maintenance were prepared using standard methods. SGalR and SRGly were identical to SD except that they contained $1 \%$ galactose plus $1 \%$ raffinose, and $2 \%$ galactose plus $3 \%$ glycerol instead of $2 \%$ glucose, respectively. Nocodazole and $\alpha$-factor were purchased from LKT Laboratories (St. Paul, MN, USA) and Genenet (Fukuoka, Japan), respectively. 
Table 1 Yeast strains used in this study

\begin{tabular}{|c|c|}
\hline Name (Alias) & Description (Source) \\
\hline SCU15 (W303a) & Mata ura3 his3 leu2 trp1 ade2 can1 (lab stock) \\
\hline SCU151 (bub2 $\Delta$ ) & SCU893 bub2::hphMX4 (this study) \\
\hline SCU396 (CEN-GFP) & SCU893 his3::GFP12-Lacl12-NLS::HIS3 trp1::LacOx256-TRP1 (this study) \\
\hline SCU397 (bub2 $\triangle$ CEN-GFP) & SCU396 bub2::/oxP (this study) \\
\hline SCU398 (CEN-GFP) & SCU893 ura3::tetO2x112::URA3 leu2::tetR-GFP-NLS::LEU2 (this study) \\
\hline SCU399 (bub2 $\triangle$ CEN-GFP) & SCU398 bub2::hphMX (this study) \\
\hline SCU404 (bub2 $\triangle$ GAL-SCC1-RRDD CEN-GFP) & SCU397 leu2:::GAL1-SCC1-R180D/R268D-HA3::LEU2 (this study) \\
\hline SCU408 (bub2 $\triangle$ PDS1-HA3) & SCU151 PDS1-HA3::URA3 (this study) \\
\hline SCU410 (bub2 $\triangle$ MET3-CDC20 CEN-GFP) & SCU399 MET3-CDC20::TRP1 (this study) \\
\hline SCU893 (bar1 $\triangle)$ & SCU15 bar1::hisG (U. Surana) \\
\hline SCU1226 (cdh1 $\triangle$ CEN-GFP) & SCU15 ura3::tetO::URA3 leu2::tetR::LEU2 cdh1::HIS3 [63] \\
\hline SCU1228 (cdh1 $\Delta)$ & SCU15 cdh1::kanR [63] \\
\hline SCU1336 (bub2 $\operatorname{cdh} 1 \triangle$ CEN-GFP) & SCU1226 bub2::kanMX (this study) \\
\hline SCU1337 (MAD2-GFP) & SCU893 mad2::kanMX [pMAD2-GFP] (this study) \\
\hline SCU1338 (bub2 $\triangle$ MAD2-GFP) & SCU151 mad2::kanMX [pMAD2-GFP] (this study) \\
\hline SCU1700 (cdh1D CEN-GFP) & SCU1228 trp 1::LacOx256:TRP1 his3::HIS3p-GFP13-Lacl12NLS::HIS3 (this study) \\
\hline SCU2755 (PDS1-HA3) & SCU893 pds 1::PDS1-HA3.:URA3 (this study) \\
\hline SCU2834 (bub2 $\triangle$ cdh1 $\triangle$ PDS1-HA3) & SCU1336 ura3 pds1::PDS1-HA3::URA3 (this study) \\
\hline
\end{tabular}

\section{Microscope observations}

Except for Mad2-GFP-expressing cells, cells expressing GFP-tagged proteins were fixed with $70 \%$ ethanol for 30 sec. After washing with distilled water, cells were stained with 4',6-diamidino-2-phenylindole (DAPI) at $1 \mu \mathrm{g} / \mathrm{ml}$ for 15 min. For detection of weak Mad2-GFP signals, cells were not fixed with ethanol. Washed cells were viewed using an Olympus IX71-23FL/S microscope (100× objective) and a cooled charge-couple device (CCD) camera (ORCA-ER-1, Hamamatsu Photonics) connected to a Scanalytics Image Processor LuminaVision (Mitani Corp., Tokyo, Japan). For Figures 4C and 5A and Additional file 1A, a Carl Zeiss Axio Imager M1 microscope with a cooled CCD camera (Carl Zeiss AxioCam MRm) was used.

Table 2 Plasmids used in this study

\begin{tabular}{|c|c|}
\hline Name (Alias) & Description (Source) \\
\hline pSCU134 (p416GAL1) & GAL1 URA3 CEN [64] \\
\hline pSCU145 (pRS414) & TRP1 CEN [65] \\
\hline pSCU563 (pLacOx256-LEU2) & lacOx256 LEU2 integrative [26] \\
\hline pSCU564 (pGFP12-Lacl12-NLS) & CUP1pro-GFP12-Lad12-NLS HIS3 integrative [26] \\
\hline pSCU683 (ptetR-GFP) & NLS-tetR-GFP LEU2 integrative [66] \\
\hline pSCU710 (ptetO2x112) & tetO2x112 URA3 integrative [66] \\
\hline pSCU740 (pNOP1-GFP) & NOP1-HA3GFP URA3 CEN (this study) \\
\hline pSCU784 (pPDS1-HA3) & PDS1-HA3 URA3 integrative [31] \\
\hline pSCU802 (pGAL1-CDC14) & GAL-CDC14-His6 URA3 CEN [67] \\
\hline pSCU816 (pGAL-SCC1-RRDD) & GAL-SCC1-R180D/R268D-HA3 LEU2 integrative [40] \\
\hline pSCU878 (pGAL-CDH1) & GAL-CDH1-GFP TRP1 CEN [68] \\
\hline pSCU896 (pGAL1-BFA1) & GAL1-BFA1 URA3 CEN [69] \\
\hline pSCU973 (pMAD2-GFP) & MAD2-GFP URA3 CEN [70] \\
\hline pSCU985 (Ylp22-MET3-CDC20) & MET3-CDC20 TRP1 integrative (F. Uhlmann) \\
\hline pSCU1212 (p416GAL1-pds1-db) & GAL1-PDS1 with mutated D-box URA3 CEN (this study) \\
\hline pSCU1214 (p416GAL1-pds1-dkb) & GAL1-PDS1 with mutated D/KEN-box URA3 CEN (this study) \\
\hline pSCU1550 (pGAL1-MAD2) & GAL1-MAD2-His6HAZZ $2 \mu$ URA3 [71] \\
\hline pSCU1575 (pGAL1-CDH1) & GAL1-CDH1-TAP $2 \mu$ TRP1 (this study) \\
\hline pSCU1576 (pGAL1-CDC14) & GAL1-CDC14-His6HAZZ $2 \mu$ TRP1 (this study) \\
\hline pSCU1701 (pMTW1-RFP) & MTW1-DsRed.T4 CEN LEU2 (this study) \\
\hline
\end{tabular}




\section{Western blotting analysis}

Western blotting was performed as described previously [62] using an anti-hemagglutinin (HA) antibody (16B12, $\mathrm{BAbCo}$ ), anti-cyclin dependent kinase (CDK) antibody (Santa Cruz), and anti-glucose-6-phosphate dehydrogenase (G6PDH) antibody (Sigma). Femtogrow chemiluminescent substrate (Michigan Diagnostics) for horseradish peroxidase (HRP) and Can Get Signals (Toyobo, Japan) as an immunoreaction enhancer solution were used. Chemiluminescent signals were detected using an LAS3000 mini (Fuji).

\section{Additional material}

Additional file 1: Mitotic slippage of MAD2-overexpressing bub2 $\triangle$ cells. (A, B) Cells of strains SCU396 (CEN-GFP) and SCU397 (bub2 $\triangle$ CENGFP) harboring plasmid pSCU1550 (pGAL-MAD2) were released from $\alpha$ factor into the nocodazole-containing medium (time 0 ), as described in Figure 1. Kinetochore localization of Mad2-GFP was monitored and counted after $6 \mathrm{~h}$. White arrows indicate colocalized Mad2-GFP and Mtw1-RFP signals.

\section{Acknowledgements}

We thank Andrew Murray, Kim Nasmyth, Frank Uhlmann, Orna Cohen-Fix, Matthias Peter, Leland Johnston, David Morgan, Uttam Surana, Kiwon Song, Booth Quimby and Benjamin Glick for generous gifts of materials, and Hisao Moriya and Kazunari Kaizu for discussion. This research was performed in part using an instrument at the Center for Instrumental Analysis of Shizuoka University. We especially thank laboratory members of TU for helpful discussion and support.

\section{Author details}

${ }^{1}$ Faculty of Science, Shizuoka University, Shizuoka University, Shizuoka 4228529, Japan. ${ }^{2}$ Department of Molecular Biotechnology, Graduate School of Advanced Sciences of Matter, Hiroshima University, 1-3-1 Kagamiyama, Higashi-Hiroshima 739-8530, Japan

\section{Authors' contributions}

$\mathrm{KT}$ conducted the vast majority of the experiments described in this manuscript. KN performed the experiments demonstrating securin degradation in mutant strains and kinetochore localization of Mad2. SM performed the experiments demonstrating mitotic slippage in MAD2overexpressing cells. TU designed the study, supervised the work, and wrote the manuscript. M. Ueno, M. Uritani and AY contributed to design of experiments. All authors approved the final manuscript.

\section{Authors' information}

Present address of M. Ueno: Department of Molecular Biotechnology, Graduate School of Advanced Sciences of Matter, Hiroshima University, 1-3-1 Kagamiyama, Higashi-Hiroshima 739-8530, Japan

\section{Competing interests}

The authors declare that they have no competing interests.

Received: 1 September 2011 Accepted: 9 February 2012 Published: 9 February 2012

\section{References}

1. Castro A, Bernis C, Vigneron S, Labbe JC, Lorca T: The anaphase-promoting complex: a key factor in the regulation of cell cycle. Oncogene 2005, 24:314-325.

2. Manchado E, Eguren M, Malumbres M: The anaphase-promoting complex/ cyclosome (APC/C): cell-cycle-dependent and -independent functions. Biochem Soc Trans 2010, 38:65-71.
3. Peters JM: The anaphase promoting complex/cyclosome: a machine designed to destroy. Nat Rev Mol Cell Biol 2006, 7:644-656.

4. Pines J: Cubism and the cell cycle: the many faces of the APC/C. Nat Rev Mol Cell Biol 2011, 12:427-438.

5. Sullivan M, Morgan DO: Finishing mitosis, one step at a time. Nat Rev Mol Cell Biol 2007, 8:894-903.

6. Schwab M, Lutum AS, Seufert W: Yeast Hct1 is a regulator of Clb2 cyclin proteolysis. Cell 1997, 90:683-693.

7. Visintin $\mathrm{R}$, Prinz $\mathrm{S}, \mathrm{Amon} \mathrm{A}: \mathrm{CDC} 20$ and $\mathrm{CDH1}$ : a family of substratespecific activators of APC-dependent proteolysis. Science 1997, 278:460-463.

8. Simanis $\mathrm{V}$ : Events at the end of mitosis in the budding and fission yeasts. J Cell Sci 2003, 116:4263-4275.

9. Stegmeier F, Amon A: Closing mitosis: the functions of the Cdc14 phosphatase and its regulation. Annu Rev Genet 2004, 38:203-232.

10. Tan AL, Rida PC, Surana U: Essential tension and constructive destruction: the spindle checkpoint and its regulatory links with mitotic exit. Biochem J 2005, 386:1-13.

11. Bharadwaj $\mathrm{R}, \mathrm{Yu} \mathrm{H}$ : The spindle checkpoint, aneuploidy, and cancer. Oncogene 2004, 23:2016-2027.

12. Musacchio A, Salmon ED: The spindle-assembly checkpoint in space and time. Nat Rev Mol Cell Biol 2007, 8:379-393.

13. Kulukian A, Han JS, Cleveland DW: Unattached kinetochores catalyze production of an anaphase inhibitor that requires a Mad2 template to prime Cdc20 for BubR1 binding. Dev Cell 2009, 16:105-117.

14. Pinsky BA, Nelson CR, Biggins S: Protein phosphatase 1 regulates exit from the spindle checkpoint in budding yeast. Curr Biol 2009, 19:1182-1187.

15. Goto GH, Mishra A, Abdulle R, Slaughter CA, Kitagawa K: Bub1-mediated adaptation of the spindle checkpoint. PLoS Genet 2011, 7:e1001282.

16. Rieder $\mathrm{CL}$, Maiato $\mathrm{H}$ : Stuck in division or passing through: what happens when cells cannot satisfy the spindle assembly checkpoint. Dev Cell 2004 7:637-651.

17. Minn AJ, Boise LH, Thompson CB: Expression of BCl-xL and loss of $\mathrm{p} 53$ can cooperate to overcome a cell cycle checkpoint induced by mitotic spindle damage. Genes Dev 1996, 10:2621-2631.

18. Brito DA, Rieder CL: Mitotic checkpoint slippage in humans occurs via cyclin B destruction in the presence of an active checkpoint. Curr Biol 2006, 16:1194-1200.

19. Gascoigne KE, Taylor SS: Cancer cells display profound intra- and interline variation following prolonged exposure to antimitotic drugs. Cancer Cell 2008, 14:111-122.

20. Lee J, Kim JA, Margolis RL, Fotedar R: Substrate degradation by the anaphase promoting complex occurs during mitotic slippage. Cell Cycle 2010, 9:1792-1801.

21. Fry AM, Yamano $\mathrm{H}$ : APC/C-mediated degradation in early mitosis: how to avoid spindle assembly checkpoint inhibition. Cell Cycle 2006, 5:1487-1491.

22. Hayes MJ, Kimata Y, Wattam SL, Lindon C, Mao G, Yamano H, Fry AM: Early mitotic degradation of Nek2A depends on Cdc20-independent interaction with the APC/C. Nat Cell Biol 2006, 8:607-614.

23. Rossio V, Galati E, Ferrari M, Pellicioli A, Sutani T, Shirahige K, Lucchini G, Piatti S: The RSC chromatin-remodeling complex influences mitotic exit and adaptation to the spindle assembly checkpoint by controlling the Cdc14 phosphatase. I Cell Biol 2010, 191.981-997.

24. Hoyt MA, Totis L, Roberts BT: S. cerevisiae genes required for cell cycle arrest in response to loss of microtubule function. Cell 1991, 66:507-517.

25. Bardin AJ, Amon A: Men and sin: what's the difference? Nat Rev Mol Cell Biol 2001, 2:815-826.

26. Straight AF, Belmont AS, Robinett CC, Murray AW: GFP tagging of budding yeast chromosomes reveals that protein-protein interactions can mediate sister chromatid cohesion. Curr Biol 1996, 6:1599-1608.

27. Fraschini R, Formenti E, Lucchini G, Piatti S: Budding yeast Bub2 is localized at spindle pole bodies and activates the mitotic checkpoint via a different pathway from Mad2. J Cell Biol 1999, 145:979-991.

28. Krishnan R, Pangilinan F, Lee C, Spencer F: Saccharomyces cerevisiae BUB2 prevents mitotic exit in response to both spindle and kinetochore damage. Genetics 2000, 156:489-500.

29. Shirayama M, Zachariae W, Ciosk R, Nasmyth K: The Polo-like kinase Cdc5p and the WD-repeat protein $\mathrm{Cdc20p/fizzy}$ are regulators and substrates of the anaphase promoting complex in Saccharomyces cerevisiae. EMBO J 1998, 17:1336-1349. 
30. Tavormina PA, Burke DJ: Cell cycle arrest in cdc20 mutants of Saccharomyces cerevisiae is independent of $\mathrm{Ndc10p}$ and kinetochore function but requires a subset of spindle checkpoint genes. Genetics 1998, 148:1701-1713

31. Cohen-Fix O, Peters JM, Kirschner MW, Koshland D: Anaphase initiation in Saccharomyces cerevisiae is controlled by the APC-dependent degradation of the anaphase inhibitor Pds1p. Genes Dev 1996, 10:3081-3093.

32. Holt $\sqcup$, Krutchinsky AN, Morgan DO: Positive feedback sharpens the anaphase switch. Nature 2008, 454:353-357.

33. Schwickart M, Havlis J, Habermann B, Bogdanova A, Camasses A, Oelschlaegel T, Shevchenko A, Zachariae W: Swm1/Apc13 is an evolutionarily conserved subunit of the anaphase-promoting complex stabilizing the association of Cdc16 and Cdc27. Mol Cell Biol 2004, 24:3562-3576.

34. Carroll CW, Enquist-Newman M, Morgan DO: The APC subunit Doc1 promotes recognition of the substrate destruction box. Curr Biol 2005, 15:11-18.

35. Nasmyth K: Segregating sister genomes: the molecular biology of chromosome separation. Science 2002, 297:559-565.

36. Meluh PB, Strunnikov AV: Beyond the ABCs of CKC and SCC. Do centromeres orchestrate sister chromatid cohesion or vice versa? Eur J Biochem 2002, 269:2300-2314.

37. Uhlmann F: Chromosome cohesion and separation: from men and molecules. Curr Biol 2003, 13:R104-114.

38. Tang X, Wang Y: Pds1/Esp1-dependent and -independent sister chromatid separation in mutants defective for protein phosphatase $2 \mathrm{~A}$ Proc Natl Acad Sci USA 2006, 103:16290-16295.

39. Renshaw MJ, Ward JJ, Kanemaki M, Natsume K, Nedelec FJ, Tanaka TU: Condensins promote chromosome recoiling during early anaphase to complete sister chromatid separation. Dev Cell 2010, 19:232-244.

40. Uhlmann F, Lottspeich F, Nasmyth K: Sister-chromatid separation at anaphase onset is promoted by cleavage of the cohesin subunit Scc1. Nature 1999, 400:37-42.

41. D'Amours D, Amon A: At the interface between signaling and executing anaphase-Cdc14 and the FEAR network. Genes Dev 2004, 18:2581-2595.

42. Strunnikov AV: A case of selfish nucleolar segregation. Cell Cycle 2005, 4:113-117.

43. Torres-Rosell J, Machin F, Aragon L: Cdc14 and the temporal coordination between mitotic exit and chromosome segregation. Cell Cycle 2005, 4:109-112.

44. de Almeida A, Raccurt I, Peyrol S, Charbonneau M: The Saccharomyces cerevisiae $\mathrm{Cdc} 14$ phosphatase is implicated in the structural organization of the nucleolus. Biol Cell 1999, 91:649-663.

45. Shirayama M, Toth A, Galova M, Nasmyth K: APC(Cdc20) promotes exit from mitosis by destroying the anaphase inhibitor Pds1 and cyclin Clb5. Nature 1999, 402:203-207.

46. Pines J: Mitosis: a matter of getting rid of the right protein at the right time. Trends Cell Biol 2006, 16:55-63.

47. van Leuken $R$, Clijsters $L$, Wolthuis R: To cell cycle, swing the APC/C. Biochim Biophys Acta 2008, 1786:49-59.

48. Wasch R, Robbins JA, Cross FR: The emerging role of $A P C / C C d h 1$ in controlling differentiation, genomic stability and tumor suppression. Oncogene 2010, 29:1-10.

49. Rudner AD, Hardwick KG, Murray AW: Cdc28 activates exit from mitosis in budding yeast. J Cell Biol 2000, 149:1361-1376.

50. Stevenson LF, Kennedy BK, Harlow E: A large-scale overexpression screen in Saccharomyces cerevisiae identifies previously uncharacterized cell cycle genes. Proc Natl Acad Sci USA 2001, 98:3946-3951.

51. Crasta K, Huang $P$, Morgan G, Winey M, Surana U: Cdk1 regulates centrosome separation by restraining proteolysis of microtubuleassociated proteins. EMBO J 2006, 25:2551-2563.

52. Crasta K, Lim HH, Giddings TH Jr, Winey M, Surana U: Inactivation of Cdh1 by synergistic action of $\mathrm{Cdk} 1$ and polo kinase is necessary for proper assembly of the mitotic spindle. Nat Cell Biol 2008, 10:665-675.

53. Krapp A, Gulli MP, Simanis V: SIN and the art of splitting the fission yeast cell. Curr Biol 2004, 14:R722-730

54. Krapp A, Simanis $\mathrm{V}$ : An overview of the fission yeast septation initiation network (SIN). Biochem Soc Trans 2008, 36:411-415.
55. Cerutti L, Simanis V: Asymmetry of the spindle pole bodies and spg1p GAP segregation during mitosis in fission yeast. J Cell Sci 1999, 112:2313-2321

56. Mulvihill DP, Hyams JS: Cytokinetic actomyosin ring formation and septation in fission yeast are dependent on the full recruitment of the polo-like kinase Plo1 to the spindle pole body and a functional spindle assembly checkpoint. J Cell Sci 2002, 115:3575-3586.

57. Liu J, Tang X, Wang H, Oliferenko S, Balasubramanian MK: The localization of the integral membrane protein Cps1p to the cell division site is dependent on the actomyosin ring and the septation-inducing network in Schizosaccharomyces pombe. Mol Biol Cell 2002, 13:989-1000.

58. Pesin JA, Orr-Weaver TL: Regulation of APC/C activators in mitosis and meiosis. Annu Rev Cell Dev Biol 2008, 24:475-499.

59. Zur A, Brandeis M: Securin degradation is mediated by fzy and fzr, and is required for complete chromatid separation but not for cytokinesis. EMBO J 2001, 20:792-801.

60. Hagting A, Den Elzen N, Vodermaier HC, Waizenegger IC, Peters JM, Pines J: Human securin proteolysis is controlled by the spindle checkpoint and reveals when the APC/C switches from activation by $\mathrm{Cdc} 20$ to $\mathrm{Cdh} 1$. J Cell Biol 2002, 157:1125-1137.

61. Jeganathan KB, Malureanu L, van Deursen JM: The Rae1-Nup98 complex prevents aneuploidy by inhibiting securin degradation. Nature 2005, 438:1036-1039.

62. Honma Y, Kitamura A, Shioda R, Maruyama H, Ozaki K, Oda Y, Mini T, Jeno $P$, Maki Y, Yonezawa K, Hurt E, Ueno M, Uritani M, Hall MN, Ushimaru T: TOR regulates late steps of ribosome maturation in the nucleoplasm via Nog1 in response to nutrients. EMBO J 2006 25:3832-3842.

63. Ross $\mathrm{KE}$, Cohen-Fix $\mathrm{O}$ : The role of Cdh1p in maintaining genomic stability in budding yeast. Genetics 2003, 165:489-503.

64. Mumberg D, Muller R, Funk M: Regulatable promoters of Saccharomyces cerevisiae: comparison of transcriptional activity and their use for heterologous expression. Nucleic Acids Res 1994, 22:5767-5768.

65. Sikorski RS, Hieter P: A system of shuttle vectors and yeast host strains designed for efficient manipulation of DNA in Saccharomyces cerevisiae. Genetics 1989, 122:19-27.

66. Michaelis C, Ciosk R, Nasmyth K: Cohesins: chromosomal proteins that prevent premature separation of sister chromatids. Cell 1997, 91:35-45

67. Jensen S, Geymonat M, Johnson AL, Segal M, Johnston LH: Spatial regulation of the guanine nucleotide exchange factor Lte1 in Saccharomyces cerevisiae. J Cell Sci 2002, 115:4977-4991.

68. Jaquenoud M, van Drogen F, Peter M: Cell cycle-dependent nuclear export of Cdh1p may contribute to the inactivation of $A P C / C(C d h 1)$. EMBO J 2002, 21:6515-6526.

69. Lee J, Hwang HS, Kim J, Song K: Ibd1p, a possible spindle pole body associated protein, regulates nuclear division and bud separation in Saccharomyces cerevisiae. Biochim Biophys Acta 1999, 1449:239-253.

70. Quimby BB, Arnaoutov A, Dasso M: Ran GTPase regulates Mad2 localization to the nuclear pore complex. Eukaryot Cell 2005, 4:274-280.

71. Gelperin DM, White MA, Wilkinson ML, Kon Y, Kung LA, Wise KJ, LopezHoyo N, Jiang L, Piccirillo SYuH, Gerstein M, Dumont ME, Phizicky EM, Snyder M, Grayhack EJ: Biochemical and genetic analysis of the yeast proteome with a movable ORF collection. Genes Dev 2005, 19:2816-2826.

doi:10.1186/1747-1028-7-4

Cite this article as: Toda et al:: APC/C-Cdh1-dependent anaphase and telophase progression during mitotic slippage. Cell Division 2012 7:4. 